\title{
Loss of UHRF2 expression is associated with human neoplasia, promoter hypermethylation, decreased 5-hydroxymethylcytosine, and high proliferative activity
}

\author{
Huarui Lu${ }^{1}$, Sweta Bhoopatiraju ${ }^{1}$, Hongbo Wang ${ }^{1}$, Nolan P. Schmitz ${ }^{1}$, Xiaohong \\ Wang $^{1}$, Matthew J. Freeman ${ }^{1}$, Colleen L. Forster ${ }^{2}$, Michael R. Verneris ${ }^{1}$, Michael \\ A. Linden ${ }^{3}$, Timothy C. Hallstrom ${ }^{1}$ \\ ${ }^{1}$ Department of Pediatrics, University of Minnesota, Minneapolis, MN 55455, USA \\ ${ }^{2}$ BioNet, Academic Health Center, University of Minnesota, Minneapolis, MN 55455, USA \\ ${ }^{3}$ Department of Laboratory Medicine and Pathology, University of Minnesota, Minneapolis, MN 55455, USA \\ Correspondence to: Timothy C. Hallstrom, email: halls026@umn.edu \\ Keywords: ubiquitin-like with PHD and ring finger domains 2, UHRF2, 5-hydroxymethylcytosine, UHRF1, leukemia \\ Received: January 08, 2016 \\ Accepted: September 24, 2016 \\ Published: October 12, 2016
}

\section{ABSTRACT}

Ubiquitin-like with PHD and ring finger domains 2 (UHRF2) binds to 5-hydroxymethylcytosine $(5 \mathrm{hmC})$, a DNA base involved in tissue development, but it is unknown how their distribution compares with each other in normal and malignant human tissues. We used IHC on human tumor specimens (160 from 19 tumor types) or normal tissue to determine the expression and distribution of UHRF2, $\mathrm{Ki}-67$, and $5 \mathrm{hmC}$. We also examined UHRF 2 expression in cord blood progenitors and compared its expression to methylation status in 6 leukemia cell lines and 15 primary human leukemias. UHRF2 is highly expressed, paralleling that of $5 \mathrm{hmC}$, in most nonneoplastic, differentiated tissue with low Ki-67 defined proliferative activity. UHRF2 is expressed in common lymphoid progenitors and mature lymphocytes but not common myeloid progenitors or monocytes. In contrast, UHRF2 immunostaining in human cancer tissues revealed widespread reduction or abnormal cytoplasmic localization which correlated with a higher $\mathrm{Ki}-67$ and reduced $5 \mathrm{hmC}$. UHRF2 expression is reduced in some leukemia cell lines, this correlates with promoter hypermethylation, and similar UHRF2 methylation profiles are seen in primary human leukemia samples. Thus, UHRF 2 and $5 \mathrm{hmC}$ are widely present in differentiated human tissues, and UHRF2 protein is poorly expressed or mislocalized in diverse human cancers.

\section{INTRODUCTION}

Epigenetic changes to DNA and histones control stem cell differentiation and the production of all types of the body's cells, and these processes often malfunction in human cancer. Methylation of cytosine (C) by DNA methyltransferase (DNMT) enzymes generates 5-methylcytosine $(5 \mathrm{mC})$ which is essential for transcriptional regulation and development. Cytosine methylation occurs most often at $\mathrm{CpG}$ dinucleotides, which form $\mathrm{CpG}$ islands (CGIs) when present together at very high density and which are often present in gene promoters. Methylation at CGIs is most often associated with gene silencing whereas demethylation of $5 \mathrm{mC}$ often leads to gene expression. $5 \mathrm{mC}$ marks can be converted to 5-hydroxymethylcytosine $(5 \mathrm{hmC})$ by the Ten-Eleventranslocation-2 (TET2) enzyme $[1,2]$. This discovery has generated great interest because TET enzymes are frequently mutated in cancer, and the $5 \mathrm{hmC}$ mark is well documented as being lost in different cancer types relative to normal tissue $[3,4,5,6]$. It is also becoming widely clear that $5 \mathrm{hmC}$ is involved in gene expression during tissue development $[7,8]$.

The UHRF2 protein was recently identified as having stronger binding affinity toward $5 \mathrm{hmC}$ than to $\mathrm{C}$ or $5 \mathrm{mC}$, and independent biochemical analysis confirmed this finding $[9,10]$. UHRF2 contains multiple domains, including a set- and ring- associated "SRA" domain that 
binds 5-hydroxymethylcytosine (5hmC), and a C-terminal Ring domain with E3 ubiquitin ligase activity [11-13]. By contrast, the SRA domain of the highly similar UHRF1 protein preferentially binds hemi-methylated DNA during replication and recruits DNMT1 to conserve methylation in the daughter strand [14-17]. UHRF2 and 5hmC were recently shown to co-localize at DNA by ChIP-seq analysis [18].

UHRF2 is a potential tumor suppressor gene located within the 9p24 chromosomal region which is among the most frequent sites of DNA copy number lost in human cancer $[19,20]$. UHRF2 was one of 100 "candidate cancer (CAN) genes" mutated in breast or colorectal cancer at a higher frequency than background mutation frequency $[21,22]$. UHRF2 has also been recovered by different research groups from diverse sleeping-beauty mutagenesis screens wherein its deletion promotes liver, colon, nervous system tumors and notably colorectal cancers with severe disease [23-25]. UHRF2 also controls gene expression relating to epithelial-mesenchymal transition and promotes cell invasion [26].

To learn more about the link between UHRF2 and $5 \mathrm{hmC}$ co-expression, we performed IHC using antibodies to compare their expression across a variety of normal and malignant human tissues. These analyses have generated several conclusions. First, UHRF2 and $5 \mathrm{hmC}$ are widely present in terminally differentiated cells. Second, UHRF2 protein levels are significantly reduced or mislocalized in a wide variety of human cancers compared to normal tissue. Finally, the UHRF2 promoter is heavily methylated in several leukemia cell lines and this correlates with reduced expression and similar methylation is also observed in human leukemia samples.

\section{RESULTS}

We used immunohistochemistry (IHC) to examine the expression pattern of UHRF2 in a large number of different normal or malignant adult human tissues. We carefully validated the UHRF2 antibody for IHC using cell line FFPE blocks (Supplementary Figure S1) using cell lines that over- or under-express UHRF2. U2OS cells with wild-type UHRF2 stain strongly but the shUHRF2 cells do not. We also performed IHC on adjacently sectioned tissues with antibodies that recognize proliferating cells (Ki67) or the $5 \mathrm{hmC}$ base. This allowed us to determine if UHRF2 is expressed in differentiated and/or proliferating normal tissue and if it is expressed in or absent from $\mathrm{Ki} 67$ positive regions of tumor sections. The $5 \mathrm{hmC}$ antibody has been extensively validated for IHC use with formalin-fixed paraffin embedded (FFPE) tissues [3, 4, 27, 28]. Normal tissue was arranged into tissue microarrays (TMAs) so that staining with anti-UHRF2 antibodies was conducted simultaneously on normal, and tumor tissues. We first evaluated UHRF2 expression patterns in normal human tissues. Results shown are representative of at least three individual tissue stainings.

We evaluated the UHRF2 staining pattern in normal, non-neoplastic adult differentiated tissue from kidney, liver, lung, pancreas, and prostate (Figure 1). Serially sectioned tissue was also processed for IHC using antibodies to detect $\mathrm{Ki} 67$ and $5 \mathrm{hmC}$. The UHRF2 protein was detectable by IHC in each of these differentiated tissues, although staining is weaker in the lung and prostate than in kidney, liver, and pancreas. UHRF2 is generally present in both the nuclear and cytoplasmic compartments of these tissues. These tissues were all negative for Ki67 staining, suggesting a low proliferative activity. In contrast, they were each strongly positive for $5 \mathrm{hmC}$ staining. Staining patterns from many other adult differentiated tissues are listed in Table 1 and shown in Supplementary Figure S2A \& S2B. An identical staining profile (UHRF2 ${ }^{+}, \mathrm{Ki}^{-} 7^{-}, 5 \mathrm{hmc}^{+}$) was observed for numerous other adult differentiated non-neoplastic tissues, such as epididymis, fallopian tube, gallbladder, skeletal muscle, parathyroid, salivary gland, seminal vesicle, and thyroid. Thus, terminally differentiated cells in many organs, some of which have previously been demonstrated to be $\mathrm{Ki} 67^{-} / 5 \mathrm{hmC}^{+}$, are generally all also positive for UHRF2.

Our staining of numerous different adult tissues also included several which contained $\mathrm{Ki} 67^{+}$progenitor cells in the process of differentiating. For example, UHRF2 strongly stained the germinal centers (GCs) of peripheral lymphoid organs including the tonsil (Figure 2), appendix and spleen (Supplementary Figure S3A). In contrast to adult differentiated tissues, which were all UHRF2 ${ }^{+}, \mathrm{Ki}^{-} 7^{-}$, $5 \mathrm{hmC}^{+}$, UHRF2 positive GCs were strongly Ki67 positive and $5 \mathrm{hmC}$ negative. The loss of $5 \mathrm{hmC}$ in GCs profile has been documented [29-31]. Intriguingly, the promoter region of the UHRF2 gene transitions from highly methylated in naïve B-lymphocytes to unmethylated in B-lymphocytes undergoing the GC reaction, consistent with the expression pattern we observe and suggestive of a potential role of UHRF2 in differentiating B-lymphocytes [32]. It also indicates that UHRF2 expression is not limited to terminally differentiated cells. We also observed UHRF2 expression in basal and suprabasal squamous epithelial cells of the oral mucosa (Figure 2), esophageal mucosa, skin, cervix and vagina (Table 1 and Supplementary Figure S3B). UHRF2 and 5hmC clearly stained Ki67 negative cells of the basal layer. Cells of the suprabasal layer were both UHRF2 positive and negative, and generally displayed strong nuclear localization of UHRF2 unlike the terminally differentiated cells which typically showed both nuclear and cytoplasmic localization. Most of these cells retained $5 \mathrm{hmC}$ in the suprabasal layer. The GI tract is a classical model of tissue differentiation where progenitor cells in the crypt produce differentiated cells of 
the villi. UHRF2 is present in both the proliferating and differentiated cells of the jejunum (Figure 2), colon, ileum, lower stomach, and rectum (Table 1 and Supplementary Figure S3C). In each of these tissue, Ki67 and 5hmC staining is mutually exclusive, with progenitor cells in the crypt $\left(\mathrm{Ki} 67^{+} / 5 \mathrm{hmC}\right)$ producing differentiated cells of the villi $\left(\mathrm{Ki}^{2} 7^{-/ 5 \mathrm{hmC}^{+}}\right)[3,31]$. IHC staining of testis revealed spermatogonia with $\mathrm{Ki}^{-} 7^{-}$nuclei that stained positive for UHRF2 and 5hmC. Differentiating spermatids displayed evidence of loss of $5 \mathrm{hmC}$ staining, acquisition of $\mathrm{Ki}^{+} 7^{+}$ staining and retention of nuclear UHRF2 staining (Figure 2D). UHRF2 also strongly stained endometrial glandular cells in the uterus, which contained $\mathrm{Ki} 67$ or $5 \mathrm{hmC}$ positive admixed cells (Figure 2E).

Having established the expression pattern of UHRF2 across numerous normal human tissues, we asked if and how the UHRF2 expression pattern is altered in human tumors originating from some of these tissues. In total, we examined 160 specimens derived from 19 different tumor types organized in tissue microarrays. This staining of tumor sample TMAs was conducted simultaneously with staining of normal tissue TMAs to reduce the likelihood of batch or slide variations in staining. This broad analysis revealed three main themes that describe the staining pattern of UHRF2 across a variety of human tumors. Some tumors retain similar expression and localization of UHRF2 compared to matching normal tissues. Second, UHRF2 appears abnormally localized to the cytoplasm but excluded from the nucleus in a subset of human tumors. Finally, UHRF2 frequently displays a significant reduction in expression in diverse tumor types. We show representative examples of each category from gastric, hepatocellular and testicular cancers.

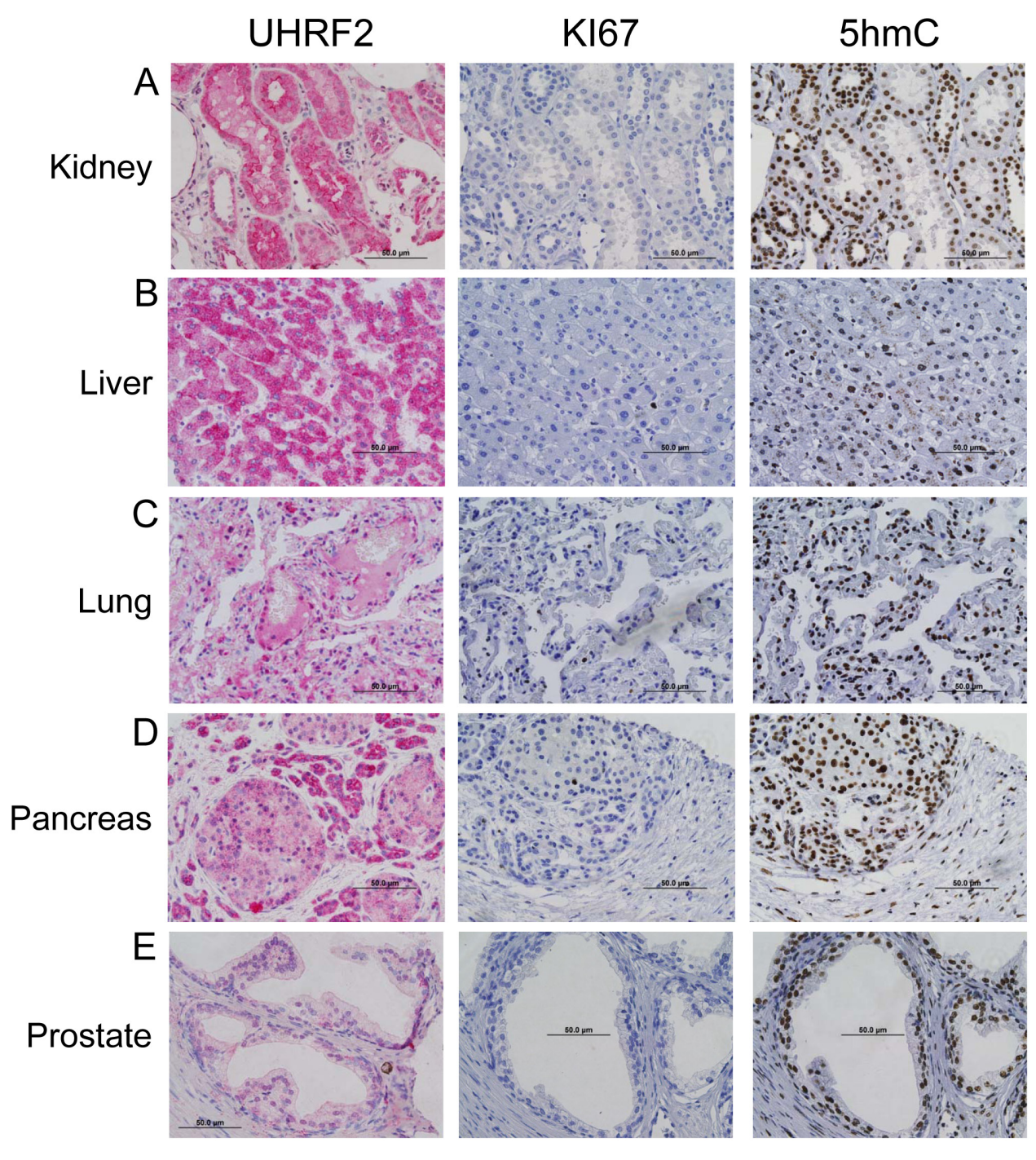

Figure 1: UHRF2 is expressed in differentiating cell populations. IHC for UHRF2, Ki67, and 5hmC was performed on normal human tissue from tonsil, oral mucosa, jejunum, testis, and uterus. 
Table 1: List of UHRF2 in different cell types of adult human tissue

\begin{tabular}{|c|c|c|}
\hline Tissue & Figure & Cell Type \\
\hline Adrenal Cortex & Supplementary Figure S2A & Fasiculata \\
\hline Appendix & Supplementary Figure S3A & Germinal center \\
\hline Cervix (Uterine) & Supplementary Figure S3B & Squamous epithelial \\
\hline Colon & Supplementary Figure S3C & Differentiating villi \\
\hline Epididymis & Supplementary Figure S2A & Some Principal \& Basal \\
\hline Esophageal Mucosa & Supplementary Figure S3B & Squamous epithelial \\
\hline Fallopian Tube & Supplementary Figure S2A & Ciliated epithelial \\
\hline Gall Bladder & Supplementary Figure S2A & Columnar epithelial \\
\hline Ilium & Supplementary Figure S3C & Differentiating villi \\
\hline Jejunum & Figure 2 & Differentiating villi \\
\hline Kidney & Figure 1 & Distal/prox convoluted tubules \\
\hline Liver & Figure 1 & Hepatocytes \\
\hline Lung & Figure 1 & Alveolar \\
\hline Oral Mucosa & Figure 2 & Squamous epithelial \\
\hline Ovary & Supplementary Figure S2B & Stroma and follicle \\
\hline Pancreas & Figure 1 & Islets of Langerhans \\
\hline Parathyroid & Supplementary Figure S2B & Chief \\
\hline Placenta & Supplementary Figure S2B & Decidual, trophoblastic, Hofbauer \\
\hline Prostate & Figure 1 & Cuboidal epithelial \\
\hline Rectum & Supplementary Figure S3C & Differentiating villi \\
\hline Salivary Gland & Supplementary Figure S2B & Interlobular ductal cells \\
\hline Seminal Vesicle & Supplementary Figure S2B & Columnar epithelial \& Lamina propria \\
\hline Skeletal Muscle & Supplementary Figure S2A & Myocytes \\
\hline Skin & Supplementary Figure S3B & Squamous epithelial \\
\hline Spleen & Supplementary Figure S3A & Germinal center \\
\hline Stomach & Supplementary Figure S3C & Differentiating villi \\
\hline Testis & Figure 2 & Spermatogonia, Spermatocytes, Sertoli \\
\hline Thyroid & Supplementary Figure S2B & Folicular \& Cuboidal epithelial \\
\hline Tonsil & Figure 2 & Germinal center \\
\hline Uterus - Endometrial & Figure 2 & Glandular epithelial \\
\hline Vagina & Supplementary Figure S3B & Squamous epithelial \\
\hline
\end{tabular}

All tissues examined by IHC for UHRF2, Ki67 and 5hmC are listed, and the UHRF2 positive cell types are listed.

UHRF2 expression was observed in the nucleus and cytoplasm of both proliferating and differentiating epithelial cells in the GI tract villi. Gastric tumors typically arise from these epithelial cells, and in some cases these tumors retain strong UHRF2 staining that is comparable to normal tissue (Figure $3 \mathrm{~A}$ ). We observed that gastric tumors also often display altered localization of UHRF2 predominantly in the cytoplasm but not the nucleus
(Figure 3B). Similar staining was observed in some colorectal tumors in this study (Supplementary Figure $\mathrm{S} 4 \mathrm{~A}$ ) and by Lu et al., who also reported cytoplasmic staining of UHRF2 in a large cohort of human colorectal tumors [33]. These gastric tumor cells were strongly $\mathrm{Ki} 67$ positive, and cells from the same region in serially sectioned tissue were notably lacking $5 \mathrm{hmC}$ staining. A final subset of gastric tumors displays a significant 
reduction in UHRF2 staining (Figure 3C). These tumors with reduced UHRF2 expression were highly $\mathrm{Ki} 7^{+}$and $5 \mathrm{hmC}$ - We assessed UHRF2 staining across a sampling of hepatic cholangiocarcinomas, which arise from biliary ductular epithelial cells. Some of these tumors retained moderate levels of UHRF2 (Figure 3D). Another subset of these cancers showed a significant increase in cytoplasmic localization of UHRF2 (Figure 3E). A final fraction of cholangiocarcinomas showed sharply reduced levels of UHRF2 (Figure 3F). Testicular cancer is thought to arise from germ cells, and these cells displayed strong nuclear UHRF2 staining in normal tissue. We show an example of a highly proliferative, $5 \mathrm{hmC}^{-}$testis cancer where UHRF2 levels are observed at moderate levels primarily in the nucleus (Supplementary Figure S4A). A second, unusual example, shows strong UHRF2 staining in a perinuclear pattern in cells that are generally $\mathrm{Ki} 67^{+}$and $5 \mathrm{hmC}$. Lastly, UHRF2 protein levels are also reduced in a highly Ki67+ testis cancer.

We sought to determine if loss of UHRF2 expression was a recurring theme in other human tumor types. We show examples of significant reduction in UHRF2 staining in pancreatic, lymphoma, cervical, endometrial, squamous cell carcinoma (SCC) and head \& neck human tumors (Figure 4). UHRF2 staining was assessed in regions that were highly proliferative in serially sectioned tissue. Highly proliferative regions $\left(\mathrm{Ki} 7^{+}\right)$almost always display reduced $5 \mathrm{hmC}$ levels by IHC staining, as seen previously in studies using human tumor samples. Occasional exceptions to this rule were noted. For example, staining

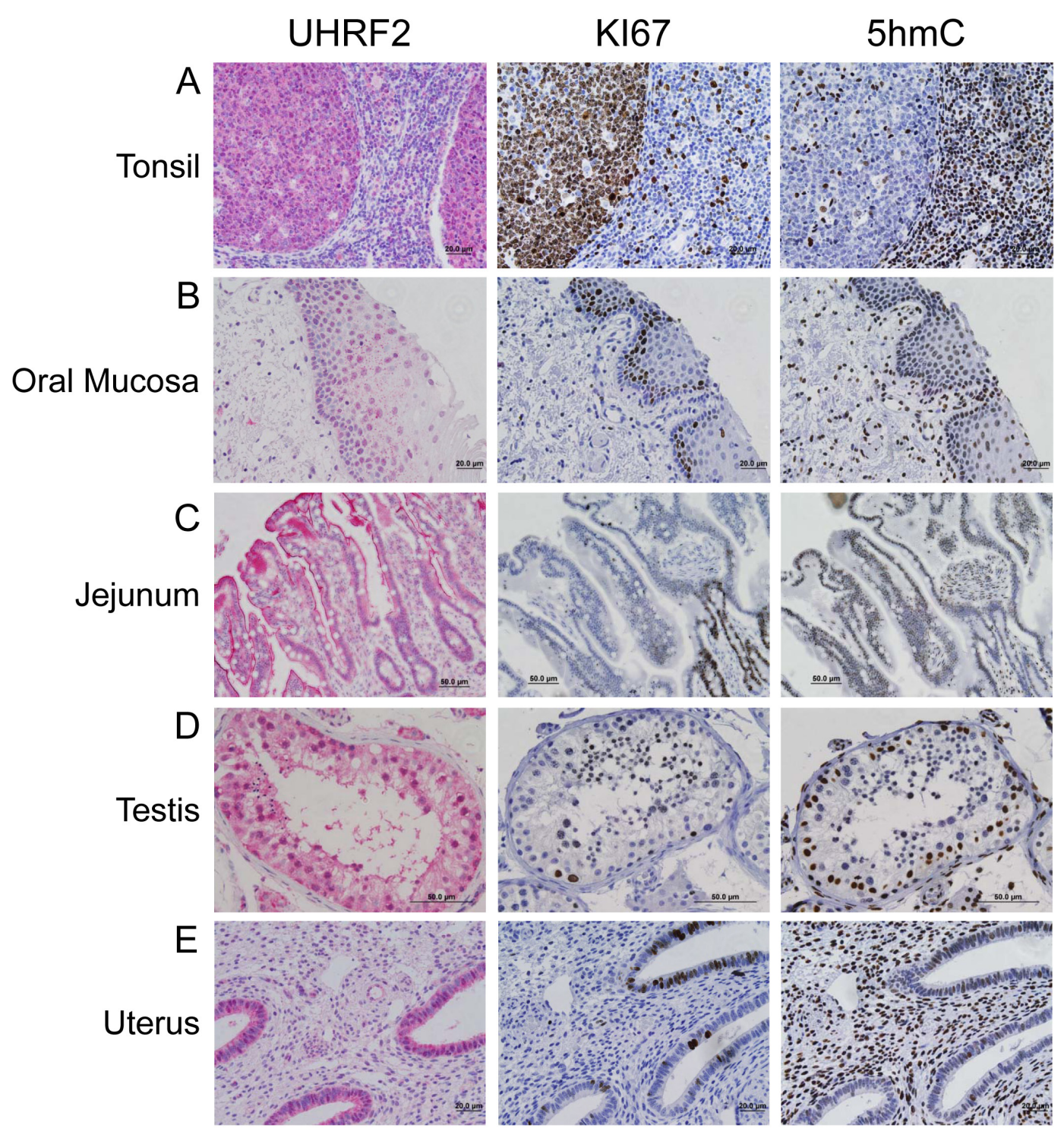

Figure 2: UHRF2 and 5hmC are hallmarks of terminally differentiated cells. Adult human tissue from kidney, liver, lung, pancreas and prostate were analyzed by IHC for UHRF2, Ki67 and 5hmC levels. 
of a testis cancer and a lung SCC (Supplementary Figure S4A) each contained $>90 \%$ cells staining positive for Ki67 and $5 \mathrm{hmC}$

This analyses documented UHRF2 expression across a variety of normal and tumor solid tissues, but had not assessed the presence of UHRF2 in normal or malignant hematological cells, so we assessed relative levels of UHRF2 in hematopoietic stem cells, progenitors or differentiated cells. First, hematopoietic stem cells (HSCs), multipotent progenitors (MPPs), common lymphoid progenitors (CLPs), and common myeloid progenitors (CMPs) were separated by fluorescent activated cell sorting (FACS) from human umbilical cord blood. RNA was isolated and quantified, and qPCR was used to measure UHRF2 mRNA levels normalized to GAPDH (Figure 5). We found that UHRF2 levels were lowest in HSCs and remained comparably low in MPPs and CMPs. Surprisingly, UHRF2 mRNA was elevated around 8-fold in CLPs compared to HSCs. Next, mature lymphocyte populations were isolated from human blood and protein analyzed by immunoblotting. We observed comparable expression of UHRF2 in isolated B- and

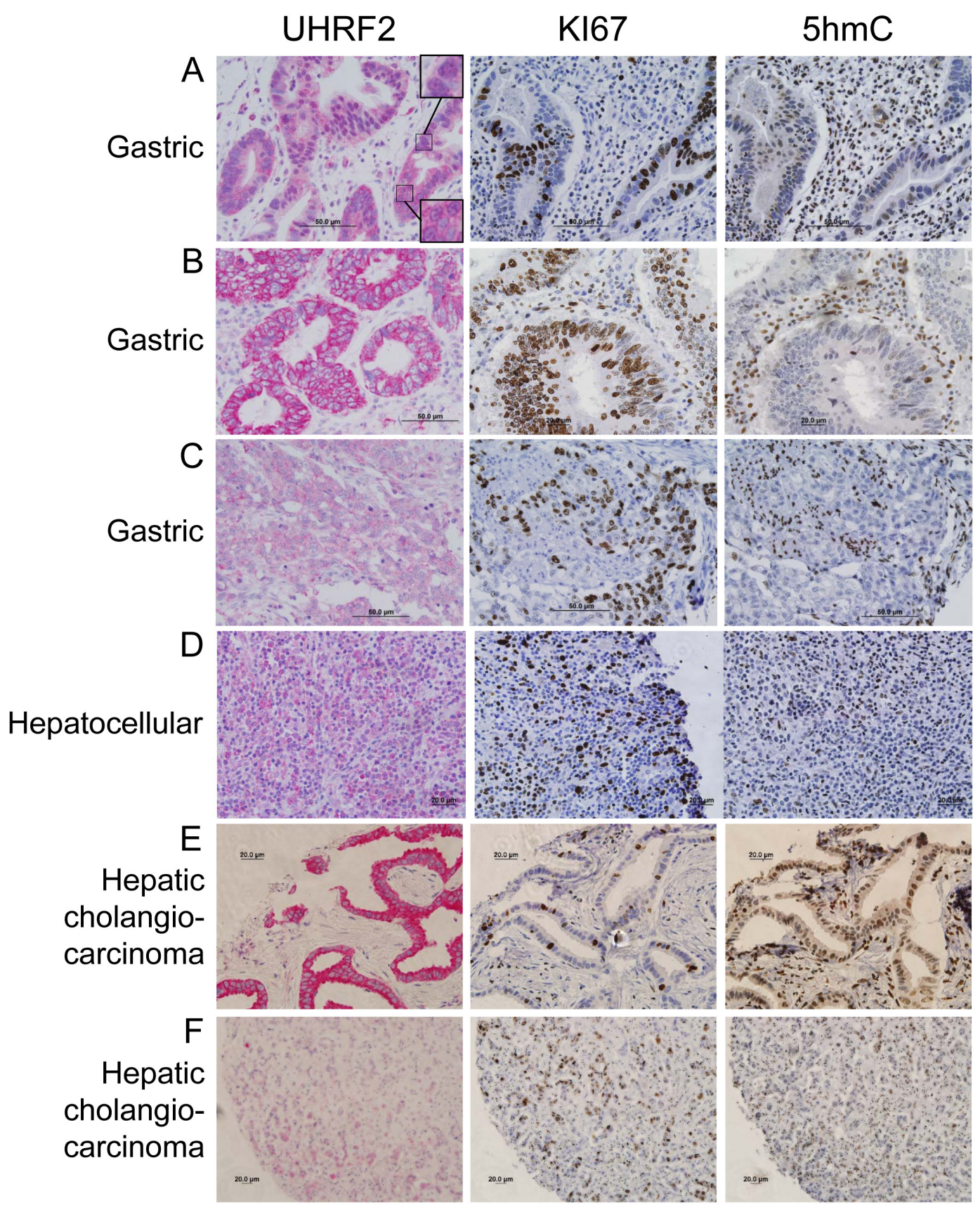

Figure 3: UHRF2 protein levels are mislocalized or reduced compared to normal tissue in gastric and hepatocellular human tumors. Human cancer tissue was analyzed by IHC for UHRF2, Ki67, and 5hmC and displayed as representative micrographs. 
T-lymphocytes. UHRF2 levels are reduced but still present in CD56+ natural killer cells. We were unable to detect UHRF2 in CD14 $4^{+}$monocytes. Thus, UHRF2 appears to be predominantly expressed in mature cells of lymphoid origin and in CLPs, but not in the more undifferentiated HSCs or MPPs, or in cells of the myeloid lineage (CMPs, monocytes).

Our previous analysis of UHRF2 expression in normal vs. cancer tissue indicated that its levels are decreased in a wide variety of human tumor types. To determine if UHRF2 is also decreased in hematopoietic cancer cells, we analyzed UHRF2 mRNA and protein expression pattern across six different leukemia or lymphoma cell lines. mRNA was isolated from Jurkat
(T-lymphoblastic leukemia), K562 (lymphoblasts), RAJI (Burkitt lymphoma), RS4:11 \& SEMK2 (t(4;11)positive precursor B-lymphoblastic leukemia), and NALM6 (B-lymphoblastic leukemia) cells and qPCR was utilized to compare UHRF2 levels across these cell lines relative to the internal control GAPDH. We found that levels of UHRF2 mRNA were comparably high in the RS4:11, SEMK2, and NALM6 cell lines but greatly reduced in Jurkat, K562 and RAJI cell lines. We saw a corresponding relationship between mRNA and protein levels from these cell lines, with Jurkat, K562 and RAJI cells not expressing UHRF2, and the RS4:11, SEMK2, and NALM6 lines highly expressing UHRF2 protein. UHRF2 protein was also reduced in U2OS cells stably

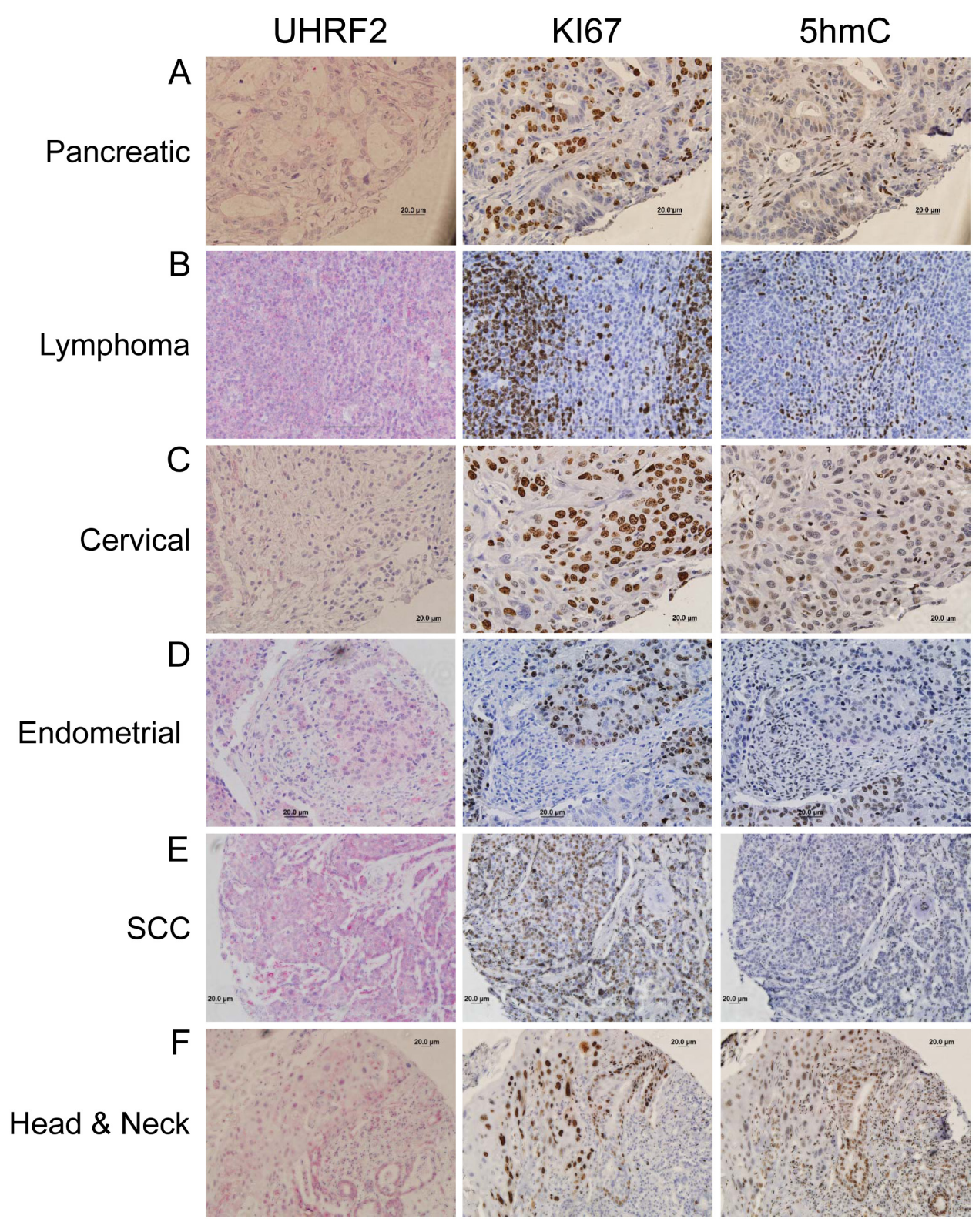

Figure 4: UHRF2 protein levels are reduced across numerous different human tumor types. Human cancer tissue was analyzed by IHC for UHRF2, Ki67, and 5hmC and displayed as representative micrographs. 
integrated with an shRNA against UHRF2, which we have previously described [34]. These findings indicate that just as for human solid tumors, UHRF2 expression is severely reduced in some hematopoietic malignancies.

Hypermethylation of CGIs in the promoter regions of genes is a frequent cause of tumor suppressor inactivation in human cancer. The UHRF2 promoter contains a 1744 nucleotide region predicted to be a $\mathrm{CpG}$ island $(65.7 \% \mathrm{GC}, 0.95 \mathrm{CpG}$ observed [Obs]/global expected [Exp]) compared to the classic definition of a $\mathrm{CpG}$ island $(\mathrm{GC}>50 \%$, Obs/Exp $>0.6)$. We thought that differential hypermethylation of the UHRF2 promoter might reasonably explain its reduced levels in human solid and hematological malignancies. We tested this directly by measuring $\mathrm{CpG}$ methylation of the UHRF2 promoter from bisulfite-treated genomic DNA isolated from two cell lines that express UHRF2 (SEMK2 \& NALM6) and two that don't (K562 and RAJI). We observed significant CpGs methylation of in the promoters of K562 and RAJI, compared to almost none in the SEMK2 and NALM6 cell lines (Figure 6A). Closer inspection of K562 and RAJI CpG methylation revealed two distinct regions of the promoter with different levels of methylation between these lines. One region, arbitrarily named "A" was almost fully methylated in both K562 and RAJI cell lines. A second region termed "B", which spanned three PCR amplification regions, was almost $100 \%$ methylated in $\mathrm{K} 562$ but the proportion of $\mathrm{CpG}$ methylation dropped to around $50 \%$ across this region in RAJI cells. A final region named "C" was unmethylated in each of the lines. Thus, loss of UHRF2 expression can be tied to promoter hypermethylation of CpGs in a leukemia and lymphoma
A

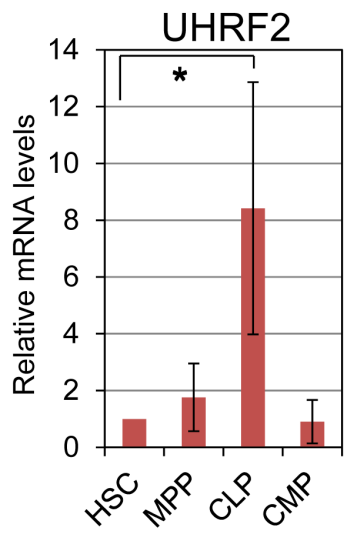

C

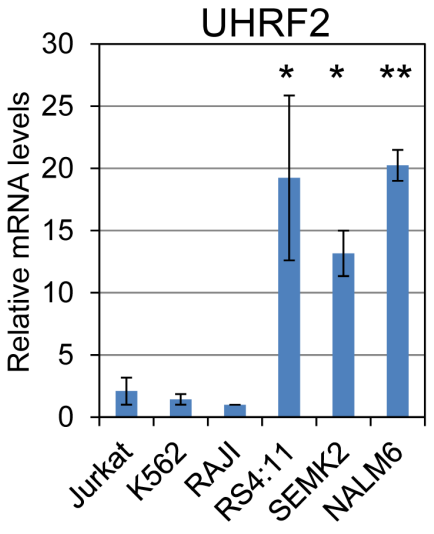

B

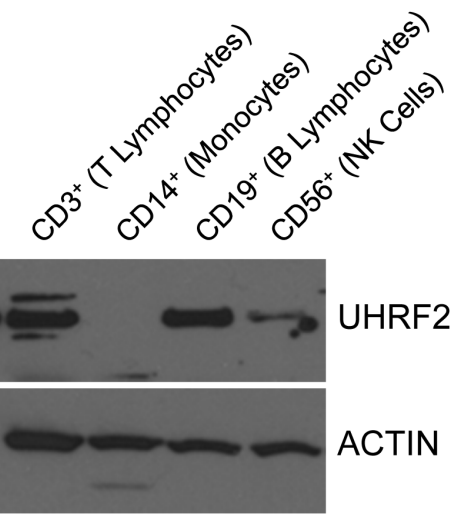

D

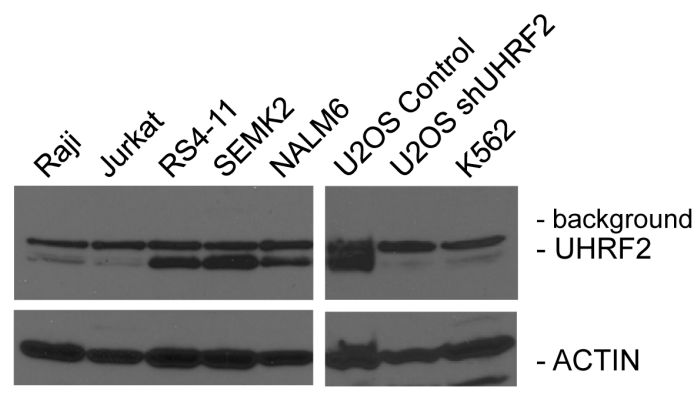

Figure 5: UHRF2 is expressed in human common lymphoid progenitors and mature B- and T- lymphocytes, and its mRNA and protein levels are substantially reduced in several human leukemia and lymphoma cells lines. A. UHRF2 mRNA expression is increased in human common lymphoid progenitors (CLP) relative to hematopoietic stem cells (HSC), multipotent progenitors (MPP) and common myeloid progenitors (CMP). $(n=3)$ B. UHRF2 protein is present in human $B$, $\mathrm{T}$, and NK cells but missing from monocytes. C. UHRF2 mRNA expression is decreased in Jurkat (T-cell leukemia), K562 (lymphoblasts) and RAJI (Burkitt's lymphoma) but intact in the pro-B and pre-B cell lines RS4:11, SEMK2, and NALM6. D. UHRF2 protein is absent in Jurkat, K562, and RAJI cell lines but present in RS4:11, SEMK2, and NALM6. See band reduced from U2OS cells containing shUHRF2 vs control. *, p < $0.05 ; * *, \mathrm{p}<0.01$. Error bars represent standard deviation from the mean. 
cell line. The $\% \mathrm{CpG}$ methylation was calculated for each region and cell line and displayed in Figure 6B.

We examined methylation of UHRF2 in primary human leukemia cells to determine the cancer relevance of these findings. We obtained 5 B-cell ALLs, 5 T-cell ALLs, and 5 AMLs with high blast counts $(>80 \%)$ and analyzed the genomic DNA by bisulfite sequencing. We used the primer set specific to the most 5 ' region of the $\mathrm{CpG}$ island, which seemed to provide the best correlation to protein expression in the cell line analysis. Each of the different tumor types displayed a unique UHRF2 methylation pattern (Figure 7A). Three of the B-cell ALLs were methylated around $25 \%$ in this region, with another showing no methylation, and a final sample at 54\% (Figure 7B). All of the T-cell ALLs were methylated across this region, averaging around $65 \%$, and thus generally much more methylated than the B-cell ALLs. Of the AML samples, three closely resembled the $65 \%$ methylation

A
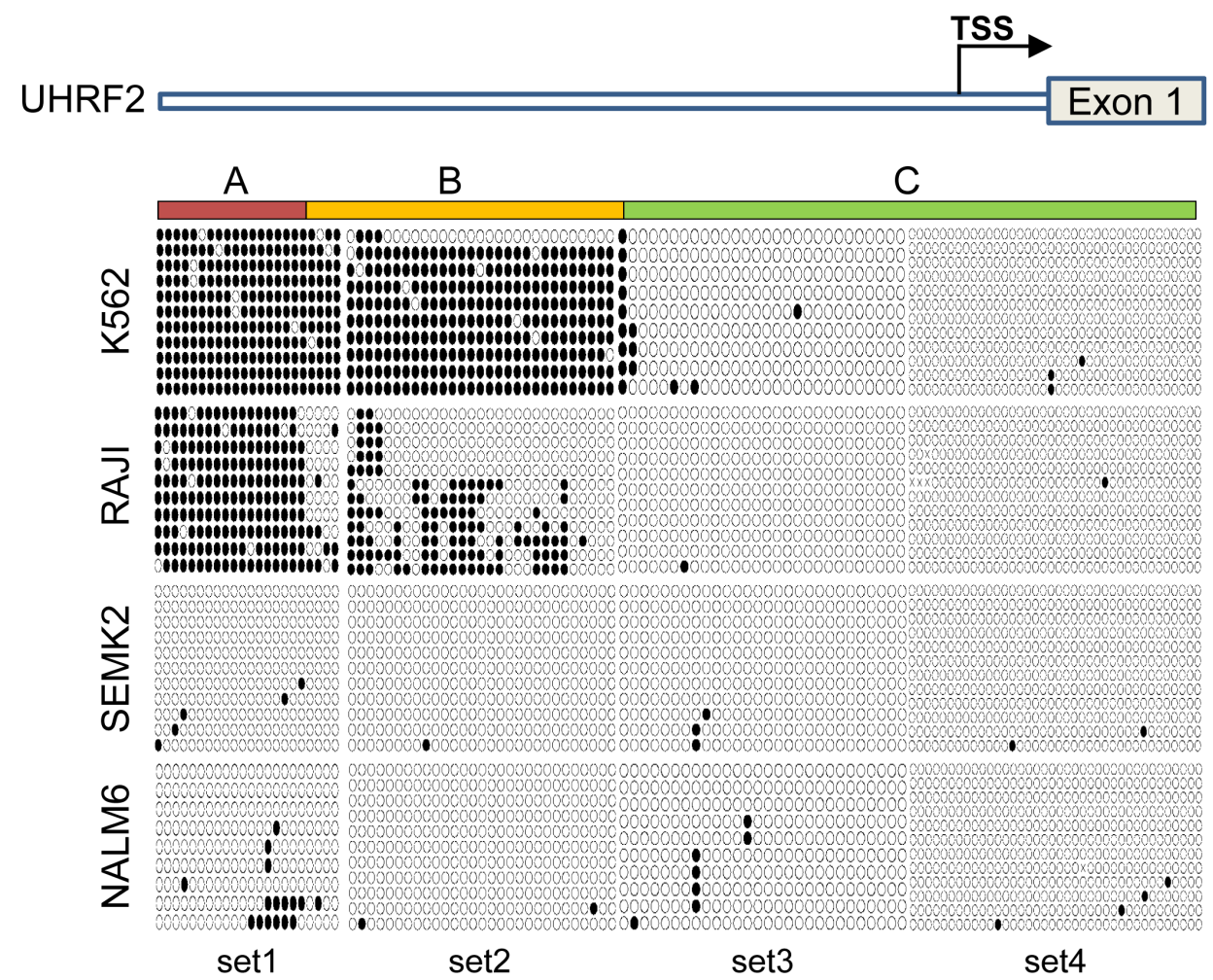

B

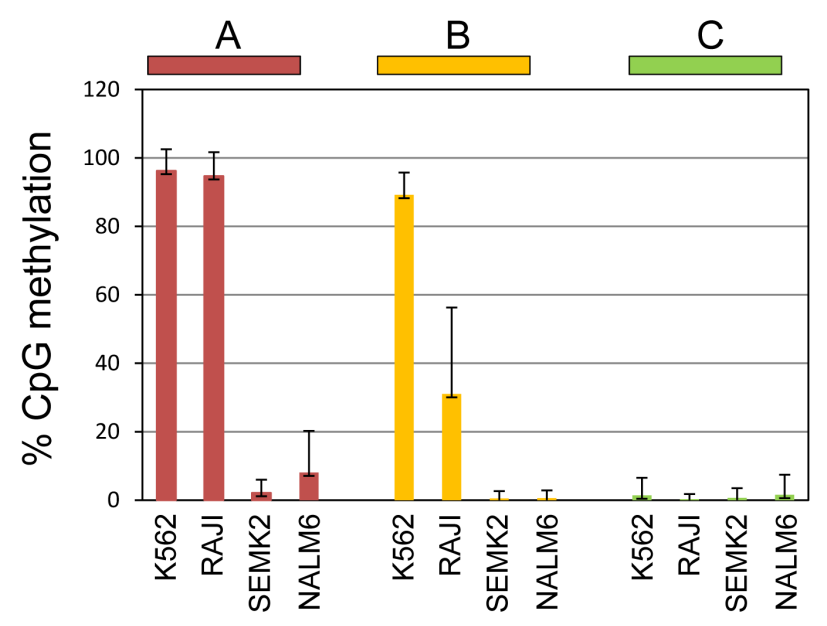

Figure 6: The UHRF2 promoter is significantly methylated in non-expressing cell lines. A. Human UHRF2 genomic locus and $\mathrm{CpGs}$ analyzed by bisulfite sequencing. Bisulfite sequencing results of individual amplicons are represented by rows of circles. Solid circles represent methylated $\mathrm{CpGs}$ and open circles denote unmethylated CpGs. B. Methylation status of UHRF2 promoter in hematopoietic cancer cell lines. The percent of CpGs methylated in regions "A", "B", and "C" were quantified and graphed. Error bars represent standard deviation from the mean. 

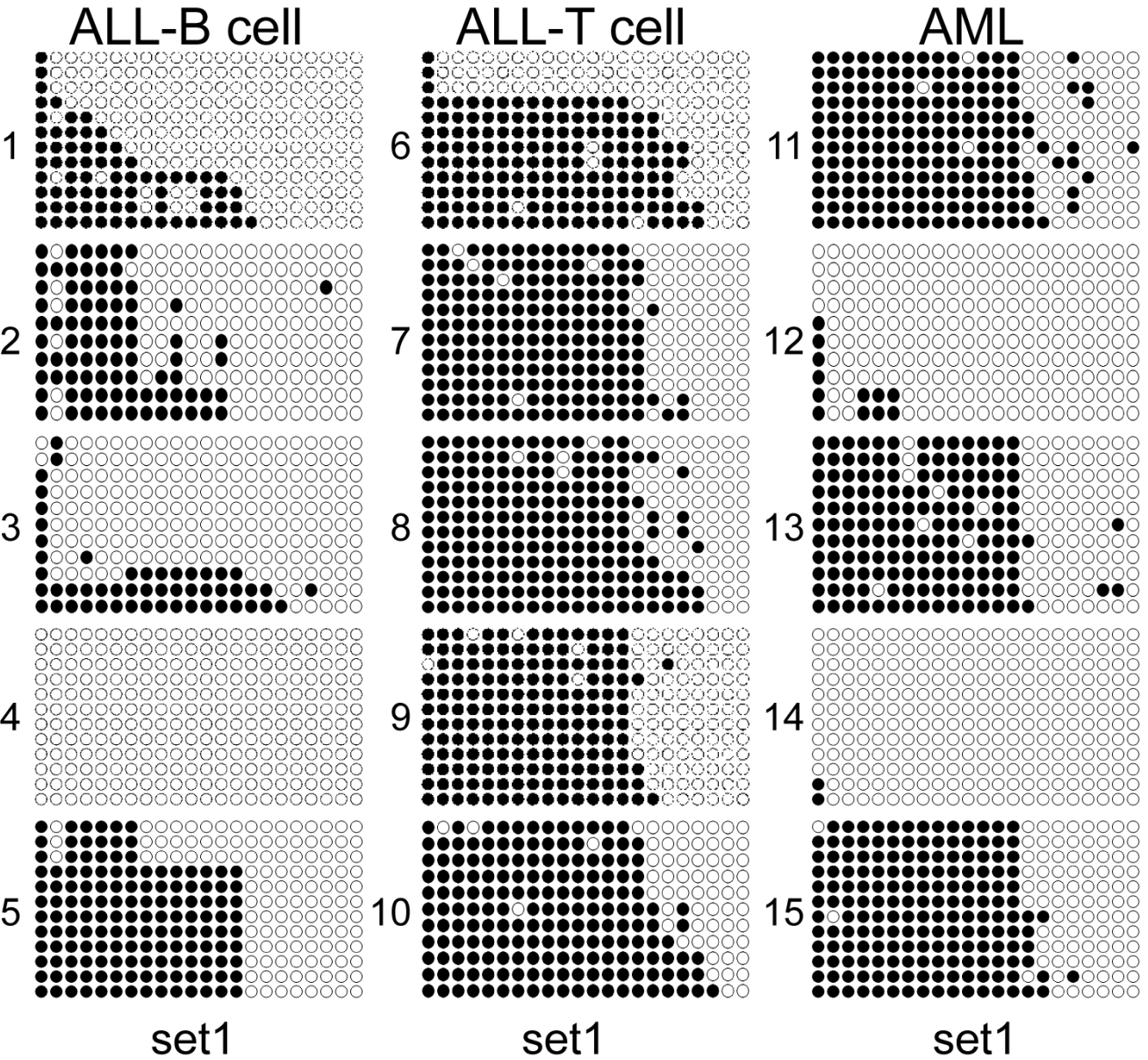

$\mathrm{B}$

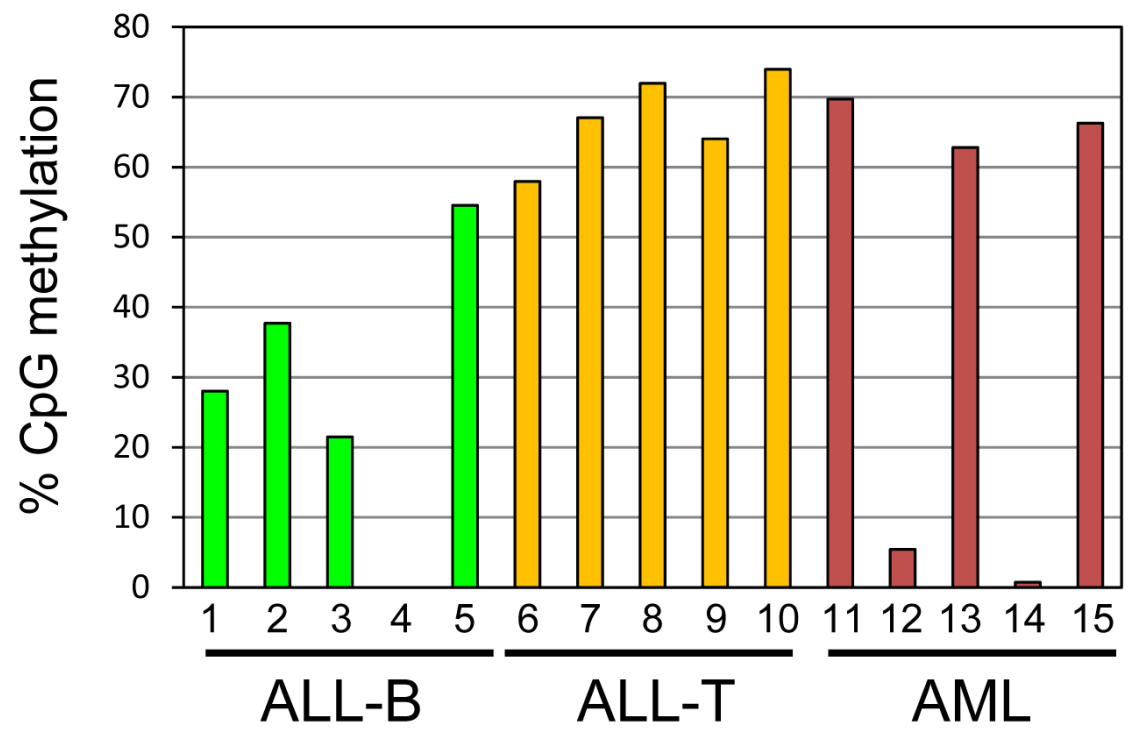

Figure 7: Variable UHRF2 methylation patterns in human leukemias. A. Bisulfite sequencing of DNA from human ALL-B cell (5), ALL-T cell (5) and AML (5). Individual CpGs are represented by solid circles (methylated) or open (unmethylated). B. The percent of CpGs methylated across this region were quantified and graphed. 
pattern of the T-cell ALLs. The remaining two samples had less than 5\% methylation. These finding indicate that the UHRF2 promoter is fairly heavily methylated in some cancer types but is greatly reduced in other types or individual cases.

We next determined if methylation patterns in the UHRF2 promoter correlated with protein expression levels in these samples. To do this, the remainder of the tumor samples were split into portions for antiUHRF2 cell staining on slides (Figure 8A), for protein immunoblotting (8B), and mRNA analysis of UHRF2 levels by qPCR (8C). Anti-UHRF2 IHC staining of cells centrifuged onto slides was validated using cell lines that express high levels (SEMK2) or low levels (K562) of UHRF2 (Supplementary Figure S5). One of the ALL-B cell leukemias stainings lost cells on the slide and was not stained. The other 4 all stained positive for UHRF2 and apparent localization at heterochromatic foci as has been described [11, 12]. All 5 ALL-B cell leukemias expressed UHRF2 by immunoblotting, indicating a good agreement with the cytospin staining. Likewise, UHRF2 mRNA was high in each of these sample compared to levels in the SEMK2 cell line. These findings indicate that this region of the UHRF2 $\mathrm{CpG}$ island is poorly methylated in $4 / 5$ ALL-B cell leukemias and this correlates with higher UHRF2 mRNA and protein levels. Among the ALL-T cell leukemias, which all were strongly methylated in this region, 2 of the 5 showed a reduction in UHRF2 protein levels by IHC and immunoblotting. However, the other samples still expressed UHRF2 protein and mRNA in spite of this region of the $\mathrm{CpG}$ island being hypermethylated. Similarly, AML samples were either fully methylated $(3 / 5)$ or unmethylated $(2 / 5)$. Of the 3 fully methylated, 2 still showed UHRF2 protein and mRNA expression. Of the 2 unmethylated, 1 showed strong UHRF2 protein and mRNA expression. These findings indicate that methylation of this region is alone insufficient to block UHRF2 expression.

\section{DISCUSSION}

UHRF2 is the most closely related and structurally similar homolog of UHRF1, and both share some overlapping and unique characteristics $[12,13]$. UHRF1 protein binds to hemi-methylated DNA during replication and recruits DNMT1 to copy the methyl pattern to the daughter strand [15-17]. Whereas the UHRF1 SRA domain shows strongest preference for hemi-methylated DNA, the SRA domain in UHRF2 show strongest biochemical affinity toward fully 5-hydroxymethylated cytosine [9, 10]. UHRF1 and UHRF2 also differ significantly in their expression patterns. UHRF1 is highly expressed in proliferating embryonic stem cells (HSCs) and a wide variety of proliferating cancer cells, but not in differentiated mature populations. In contrast, UHRF2 is not expressed in ESCs but its mRNA expression rises as ESC differentiate [12, 35].

Our results indicate that UHRF2 protein levels are widely lost in human cancer, with significant protein level reductions occurring in gastric, liver, pancreatic, lymphoma, cervical, endometrial, squamous cell carcinoma, and head and neck cancers. In gastric and liver cancers, UHRF2 appears to be preferentially localized to the cytoplasm in a subset of these tumors. A similar staining profile was observed by $\mathrm{Lu}$ and colleagues, who analyzed UHRF2 staining in a large cohort of primary colorectal tumors [33]. They observed low UHRF2 levels in $34 \%$ of tumors, and strong UHRF 2 staining in $37 \%$ of tumors, with the majority of UHRF2 localized to the cytoplasm in the strong staining tumors. The functional significance or function of cytoplasmic localized UHRF2, or what signaling events cause it to be excluded from the nucleus is unknown. However, the highly similar UHRF1 is also present in the nucleus and cytoplasm and its phosphorylation by CCNA2/CDK2 during S-phase promotes its cytoplasmic localization, and mutating the phosphor-acceptor site leads to UHRF1 nuclear accumulation [36]. Thus, altered cell cycle signaling in certain tumors may induce the cytoplasmic localization of UHRF2.

UHRF2 is altered in human tumors through a variety of mechanisms. The first, although rare, is through direct mutation in its coding sequence [21, 22]. Second, UHRF2 is located at 9p24 and is subject to DNA copy number loss in human brain, breast, gastric, kidney, hematopoietic, and lung tumors [20]. It is also overexpressed at the mRNA levels in lung adenocarcinomas and squamous cell carcinomas compared to normal tissue, although not as highly as UHRF1 or DNMTs [37]. Third, UHRF2 promoter is hypermethylated in cancer. Varley and colleagues analyzed promoter methylation of each of the 100 "CAN" genes identified as mutated in breast and colon cancer and found that UHRF2 was one of only 5 genes that was methylated in both tumor types but not normal matched tissue [38]. Our results have extended these findings by demonstrating that expression of UHRF2 mRNA is lost in cell lines with hypermethylated UHRF2. We also observe UHRF2 methylation patterns that are distinct for human T- ALL, B-ALL, and AMLs. We were unable to definitively tie methylation status of UHRF2 to its expression in human leukemia samples. However, it is important to point out that standard bisulfite sequence does not discriminate $\mathrm{mC}$ from $\mathrm{hmC}$. Thus it is possible that our tumor samples with high UHRF2 expression have hydryxomethylated promoters which might cause increased gene expression even though they were detected as hypermethylated. Together, these findings indicate that UHRF 2 and $5 \mathrm{hmC}$ are widely present in differentiated human tissues, and UHRF2 protein is poorly expressed or mislocalized in diverse human cancers. 
A

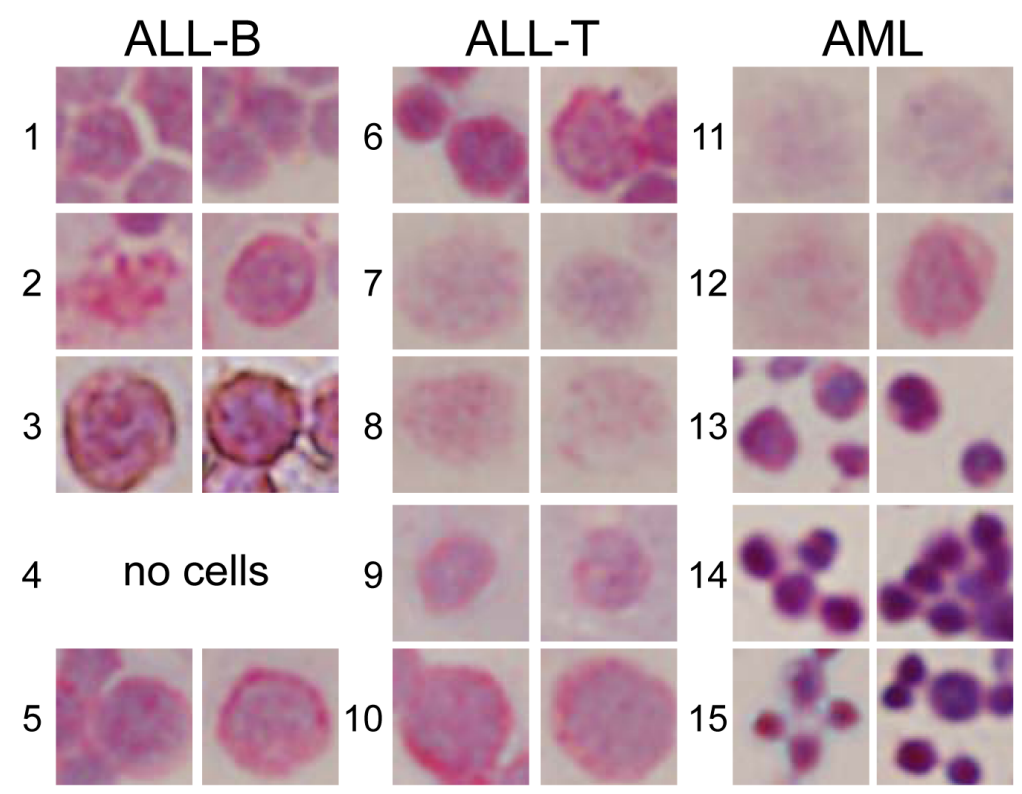

B
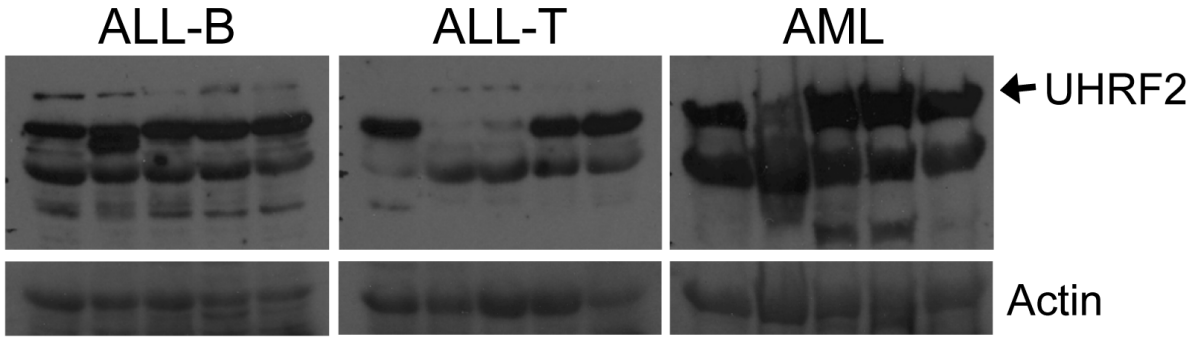

$\begin{array}{lllllllllllllll}1 & 2 & 3 & 4 & 5 & 6 & 7 & 8 & 9 & 10 & 11 & 12 & 13 & 14 & 15\end{array}$

C

UHRF2

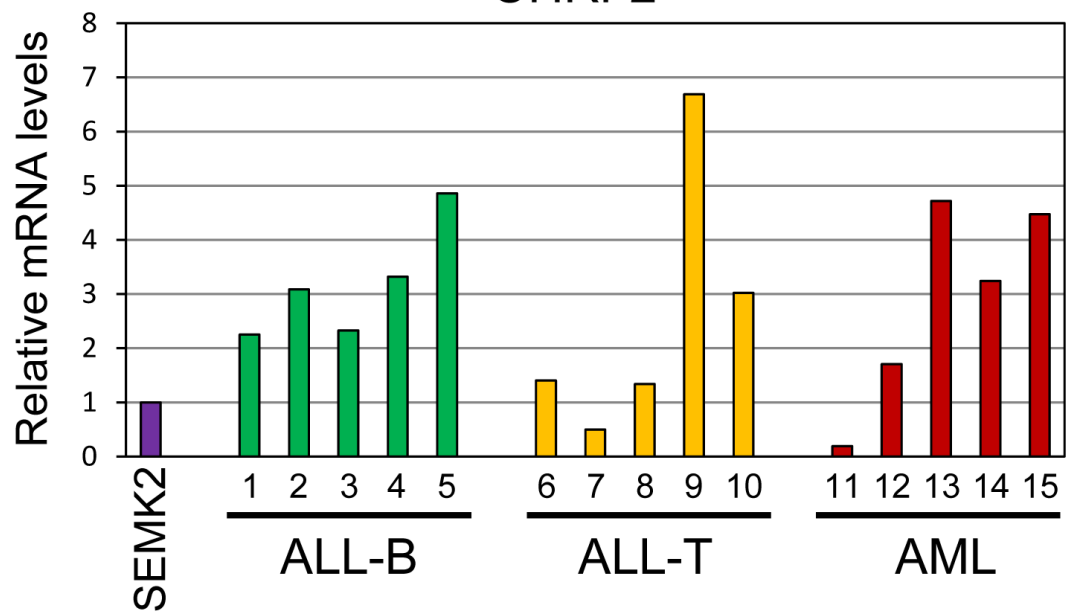

Figure 8: UHRF2 expression is altered in human leukemias. The remaining cells were split for UHRF2 expression analysis. A. Tumor cells were centrifuged onto slides, fixed, and analyzed by IHC for UHRF2 (1:2000). B. Proteins from tumor cell lysates were analyzed by SDS-PAGE and immunoblotted for UHRF2 and ACTIN. C. UHRF2 mRNA levels in tumor cells assessed by qPCR. 


\section{MATERIALS AND METHODS}

\section{TMA construction \& immunohistochemistry}

TMAs were constructed from normal and neoplastic tissues collected at the University of Minnesota Medical Center. IHC was performed as previously described [39]. Briefly, detection was through primary antibodies against UHRF2 (Sigma HPA026697/HPA026633) (1:200), 5hmC (Active Motif F3165) (1:5000), \& Ki-67 (SP6) (Biocare Medical, Cat\# CRM325), with Vector biotinylated secondary (1:250), tertiary was streptavidin-horseradish peroxidase (Covance \#SIG-32000) and chromagen 3, 3'-diaminobenzidine substrate (Covance \#SIG-31043). Micrographs were taken at 400x magnification, except where noted.

\section{Cell culture, RNA isolation, real-time PCR, and protein immunoblotting}

Cells were cultured as previously described [40]. RNA was isolated and quantitative PCR performed as described [41]. Immunoblotting was performed as described [42]. Antiserum against UHRF2 for immunoblotting was purchased from Sigma (HPA026633).

\section{Mature lymphocyte isolation}

For T, B, NK and monocyte isolation, peripheral blood was obtained from healthy donors and red blood cells and granulocytes were removed by Ficoll density centrifugation. Mononuclear cells were isolated and subjected to magnetic bead separation using T, B, NK or monocyte isolation kits (\#130-050-101, 130-092-657, 130-050-301, 130-050-201) according to the manufactures specifications (Miltenyi Biotech, Auburn, CA). Samples were $>85 \%$ pure by FACS analysis.

\section{Hematopoietic stem and progenitor cell isolation}

Umbilical cord blood mononuclear cells were isolated using Ficoll density centrifugation. CD34+ cells were isolated using magnetic bead selection according to the manufactures specification (\#130046-702, Miltenyi Biotech, Auburn, CA). Isolated cells were further purified using FACS sorting (FACS Aria I) into the following populations: HSC $(\mathrm{CD} 34+\mathrm{CD} 38-$ CD90+CD45RA-), MPP (CD34+CD38-CD90-CD45RA-), CMP (CD34+CD38+CD135+CD45RA-) and CLP $(\mathrm{CD} 34+\mathrm{CD} 10+)$. Purified cells were resuspended in RLT buffer for RNA isolation.

\section{Leukemia tumor sample analysis}

5 cases each of human B-cell ALL, T-cell ALL, and AML (blast count $>75 \%$ ) were obtained from the
University of Minnesota Leukemia MDS Tissue Bank (IRB\# 1603R86027). For cytospin analysis, 20,000 cells were spun onto a slide (10 $\mathrm{min} / 1000 \mathrm{rpm})$ using a Shandon Cytospin 3 centrifuge. Cells were fixed with $10 \%$ formalin for 20 minutes at room temperature, washed three times with PBS before analyzing by IHC using anti-UHRF2 antisera (1:2000).

\section{Bisulfite DNA conversion and sequencing method}

Genomic DNA was isolated from cell lines using QIAamp DNA Mini kit (Qiagen) according to the manufacturer's manual. Bisulfite conversion was performed using the EpiTect Fast DNA Bisulfite kit (Qiagen) following manufacturer's protocol. DNA Singlestep PCR amplification was conducted using Accuprime supermix II (Invitrogen). Primers were designed to specifically amplify converted DNA using the publicly available EpiDesigner BETA (http://www.epidesigner. com) and amplification products were visualized by agarose gel electrophoresis and appropriate bands were purified by using QIAquick Gel Extraction kit (Qiagen). Purified products were cloned into Topo TA vector for sequencing. Alignment and methylation analysis were performed using the online QUMA program (http://quma. cdb.riken.jp).

Primers for bisulfite: set1: left: GATTTTTTAGTT GTAGTAGGGAAGGA, right: ACAACTCCAAACCTA TCCTCAAAC; set2: left: GGTTTGAGGATAGGTTT GGAGTT, right:AATTCTTTAATCTCAAAAACACA CCA; set3:left:TGGTGTGTTTTTGAGATTAAAGAA TTA, right:AAAACTAAAACTCCCACATAAAAATC; set4: left:TGATTTTTATGTGGGAGTTTTAGTTTT, right:CCCTTTATCTCCCCCTAAACTCTA

\section{CONFLICTS OF INTEREST}

The authors disclose no potential conflicts of interest.

\section{GRANT SUPPORT}

This work was funded by grants to T.H. from NIH (R01CA168622), a hematological malignancy award from the Masonic Cancer Center at the University of Minnesota (T.H and M.A.L.), and the Children's Cancer Research Fund, Minneapolis, MN.

\section{REFERENCES}

1. Tahiliani M, Koh KP, Shen Y, Pastor WA, Bandukwala $\mathrm{H}$, Brudno Y, Agarwal S, Iyer LM, Liu DR, Aravind L, Rao A. Conversion of 5-methylcytosine to 5-hydroxymethylcytosine in mammalian DNA by MLL partner TET1. Science. 2009; 324:930-935. 
2. Koh KP, Rao A. DNA methylation and methylcytosine oxidation in cell fate decisions. Curr Opin Cell Biol. 2013; 25:152-161.

3. Haffner MC, Chaux A, Meeker AK, Esopi DM, Gerber J, Pellakuru LG, Toubaji A, Argani P, Iacobuzio-Donahue C, Nelson WG, Netto GJ, De Marzo AM, Yegnasubramanian S. Global 5-hydroxymethylcytosine content is significantly reduced in tissue stem/progenitor cell compartments and in human cancers. Oncotarget. 2011; 2:627-637. doi: 10.18632/oncotarget.316.

4. Yang H, Liu Y, Bai F, Zhang JY, Ma SH, Liu J, Xu ZD, Zhu HG, Ling ZQ, Ye D, Guan KL, Xiong Y. Tumor development is associated with decrease of TET gene expression and 5-methylcytosine hydroxylation. Oncogene. 2012; 32:663-669.

5. Munari E, Chaux A, Vaghasia AM, Taheri D, Karram S, Bezerra SM, Gonzalez Roibon N, Nelson WG, Yegnasubramanian S, Netto GJ, Haffner MC. Global 5-Hydroxymethylcytosine Levels Are Profoundly Reduced in Multiple Genitourinary Malignancies. PLoS One. 2016; 11:e0146302.

6. Ko M, An J, Rao A. DNA methylation and hydroxymethylation in hematologic differentiation and transformation. Curr Opin Cell Biol. 2015; 37:91-101.

7. Hon GC, Song CX, Du T, Jin F, Selvaraj S, Lee AY, Yen CA, Ye Z, Mao SQ, Wang BA, Kuan S, Edsall LE, Zhao BS, Xu GL, He C, Ren B. $5 \mathrm{mC}$ oxidation by Tet2 modulates enhancer activity and timing of transcriptome reprogramming during differentiation. Mol Cell. 2014; 56:286-297.

8. Chapman CG, Mariani CJ, Wu F, Meckel K, Butun F, Chuang A, Madzo J, Bissonette MB, Kwon JH, Godley LA. TET-catalyzed 5-hydroxymethylcytosine regulates gene expression in differentiating colonocytes and colon cancer. Sci Rep. 2015; 5:17568.

9. Spruijt CG, Gnerlich F, Smits AH, Pfaffeneder T, Jansen PW, Bauer C, Münzel M, Wagner M, Müller M, Khan F, Eberl HC, Mensinga A, Brinkman AB, et al. Dynamic readers for 5-(hydroxy)methylcytosine and its oxidized derivatives. Cell. 2013 152:1146-1159.

10. Zhou T, Xiong J, Wang M, Yang N, Wong J, Zhu B, Xu RM. Structural basis for hydroxymethylcytosine recognition by the SRA domain of UHRF2. Mol Cell. 2014; 54:879-886.

11. Zhang J, Gao Q, Li P, Liu X, Jia Y, Wu W, Li J, Dong S, Koseki H, Wong J. S phase-dependent interaction with DNMT1 dictates the role of UHRF1 but not UHRF2 in DNA methylation maintenance. Cell Res. 2011; 21:1723-1739.

12. Pichler G, Wolf P, Schmidt CS, Meilinger D, Schneider K, Frauer C, Fellinger K, Rottach A, Leonhardt H. Cooperative DNA and histone binding by Uhrf2 links the two major repressive epigenetic pathways. J Cell Biochem 2011; 112:2585-2593.
13. Mori T, Ikeda DD, Yamaguchi Y, Unoki M. NIRF/UHRF2 occupies a central position in the cell cycle network and allows coupling with the epigenetic landscape. FEBS Lett. 2012; 586:1570-1583.

14. Bostick M, Kim JK, Estève PO, Clark A, Pradhan $\mathrm{S}$, Jacobsen SE. UHRF1 plays a role in maintaining DNA methylation in mammalian cells. Science. 2007; 317:1760-1764.

15. Arita K, Ariyoshi M, Tochio H, Nakamura Y, Shirakawa M. Recognition of hemi-methylated DNA by the SRA protein UHRF1 by a base-flipping mechanism. Nature. 2008; 455:818-821.

16. Avvakumov GV, Walker JR, Xue S, Li Y, Duan S, Bronner $\mathrm{C}$, Arrowsmith $\mathrm{CH}$, Dhe-Paganon S. Structural basis for recognition of hemi-methylated DNA by the SRA domain of human UHRF1. Nature. 2008; 455:822-825.

17. Hashimoto H, Horton JR, Zhang X, Bostick M, Jacobsen SE, Cheng $X$. The SRA domain of UHRF1 flips 5-methylcytosine out of the DNA helix. Nature. 2008; 455:826-829.

18. Liu Y, Zhang B, Kuang H, Korakavi G, Lu LY, Yu X. Zinc Finger Protein 618 Regulates the Function of UHRF2 (Ubiquitin-like with PHD and Ring Finger Domains 2) as a Specific 5-Hydroxymethylcytosine Reader. J Biol Chem. 2016; 291:13679-13688.

19. Knuutila S, Aalto Y, Autio K, Bjorkqvist AM, El-Rifai W, Hemmer S, Huhta T, Kettunen E, Kiuru-Kuhlefelt S, Larramendy ML, Lushnikova T, Monni O, Pere H, et al. DNA copy number losses in human neoplasms. Am J Pathol. 1999; 155:683-694.

20. Mori T, Ikeda DD, Fukushima T, Takenoshita S, Kochi H. NIRF constitutes a nodal point in the cell cycle network and is a candidate tumor suppressor. Cell Cycle. 2011; 10:3284-3299.

21. Wood LD, Parsons DW, Jones S, Lin J, Sjoblom T, Leary RJ, Shen D, Boca SM, Barber T, Ptak J, Silliman N, Szabo $\mathrm{S}$, Dezso Z, et al. The genomic landscapes of human breast and colorectal cancers. Science. 2007; 318:1108-1113.

22. Sjoblom T, Jones S, Wood LD, Parsons DW, Lin J, Barber TD, Mandelker D, Leary RJ, Ptak J, Silliman N, Szabo S, Buckhaults P, Farrell C, et al. The consensus coding sequences of human breast and colorectal cancers. Science. 2006; 314:268-274.

23. Bard-Chapeau EA, Nguyen AT, Rust AG, Sayadi A, Lee P, Chua BQ, New LS, de Jong J, Ward JM, Chin CK, Chew $\mathrm{V}$, Toh HC, Abastado JP, et al. Transposon mutagenesis identifies genes driving hepatocellular carcinoma in a chronic hepatitis B mouse model. Nat Genet. 2013; 46:24-32.

24. March HN, Rust AG, Wright NA, ten Hoeve J, de Ridder J, Eldridge M, van der Weyden L, Berns A, Gadiot J, Uren A, Kemp R, Arends MJ, Wessels LF, Winton DJ, Adams DJ. Insertional mutagenesis identifies multiple networks of cooperating genes driving intestinal tumorigenesis. Nat Genet. 2011; 43:1202-1209. 
25. Rahrmann EP, Watson AL, Keng VW, Choi K, Moriarity BS, Beckmann DA, Wolf NK, Sarver A, Collins MH, Moertel CL, Wallace MR, Gel B, Serra E, Ratner N, Largaespada DA. Forward genetic screen for malignant peripheral nerve sheath tumor formation identifies new genes and pathways driving tumorigenesis. Nat Genet. 2013; 45:756-766.

26. Lai M, Liang L, Chen J, Qiu N, Ge S, Ji S, Shi T, Zhen B, Liu M, Ding C, Wang Y, Qin J. Multidimensional Proteomics Reveals a Role of UHRF2 in the Regulation of Epithelial-Mesenchymal Transition (EMT). Mol Cell Proteomics. 2016; 15:2263-2278.

27. Iqbal K, Jin SG, Pfeifer GP, Szabo PE. Reprogramming of the paternal genome upon fertilization involves genomewide oxidation of 5-methylcytosine. Proc Natl Acad Sci U S A. 2011; 108:3642-3647.

28. Globisch D, Munzel M, Muller M, Michalakis S, Wagner M, Koch S, Bruckl T, Biel M, Carell T. Tissue distribution of 5-hydroxymethylcytosine and search for active demethylation intermediates. PLoS One. 2011; 5:e15367.

29. Haffner MC, Pellakuru LG, Ghosh S, Lotan TL, Nelson WG, De Marzo AM, Yegnasubramanian S. Tight correlation of 5-hydroxymethylcytosine and Polycomb marks in health and disease. Cell Cycle. 2013; 12:1835-1841.

30. Matsuda I, Imai Y, Hirota S. Distinct global DNA methylation status in B-cell lymphomas: immunohistochemical study of 5-methylcytosine and 5-hydroxymethylcytosine. J Clin Exp Hematop. 2014; 54:67-73.

31. Bachman M, Uribe-Lewis S, Yang X, Williams M, Murrell A, Balasubramanian S. 5-Hydroxymethylcytosine is a predominantly stable DNA modification. Nat Chem. 2014; 6:1049-1055.

32. Shaknovich R, Cerchietti L, Tsikitas L, Kormaksson M, De S, Figueroa ME, Ballon G, Yang SN, Weinhold N, Reimers M, Clozel T, Luttrop K, Ekstrom TJ, et al. DNA methyltransferase 1 and DNA methylation patterning contribute to germinal center B-cell differentiation. Blood. 2011; 118:3559-3569.

33. Lu S, Yan D, Wu Z, Jiang T, Chen J, Yuan L, Lin J, Peng Z, Tang H. Ubiquitin-like with PHD and ring finger domains 2 is a predictor of survival and a potential therapeutic target in colon cancer. Oncol Rep. 2014; 31:1802-1810.

34. Lu H, Hallstrom TC. The nuclear protein UHRF2 is a direct target of the transcription factor E2F1 in the induction of apoptosis. J Biol Chem. 2013; 288:23833-23843.

35. Kurimoto K, Yabuta Y, Hayashi K, Ohta H, Kiyonari H, Mitani T, Moritoki Y, Kohri K, Kimura H, Yamamoto T, Katou Y, Shirahige K, Saitou M. Quantitative Dynamics of Chromatin Remodeling during Germ Cell Specification from Mouse Embryonic Stem Cells. Cell Stem Cell. 2015; 16:517-532.

36. Chu J, Loughlin EA, Gaur NA, SenBanerjee S, Jacob V, Monson C, Kent B, Oranu A, Ding Y, Ukomadu C, Sadler KC. UHRF1 phosphorylation by cyclin A2/cyclindependent kinase 2 is required for zebrafish embryogenesis. Mol Biol Cell. 2011; 23:59-70.

37. Jia Y, Li P, Fang L, Zhu H, Xu L, Cheng H, Zhang J, Li F, Feng Y, Li Y, Li J, Wang R, Du JX, et al. Negative regulation of DNMT3A de novo DNA methylation by frequently overexpressed UHRF family proteins as a mechanism for widespread DNA hypomethylation in cancer. Cell Discov. 2016; $2: 16007$.

38. Varley KE, Mitra RD. Bisulfite Patch PCR enables multiplexed sequencing of promoter methylation across cancer samples. Genome Res. 2010; 20:1279-1287.

39. Xie C, Lu H, Nomura A, Hanse EA, Forster CL, Parker JB, Linden MA, Karasch C, Hallstrom TC. Co-deleting Pten with $\mathrm{Rb}$ in retinal progenitor cells in mice results in fully penetrant bilateral retinoblastomas. Mol Cancer. 2015; 14:93.

40. $\mathrm{Lu} \mathrm{H}$, Hallstrom TC. Sensitivity to TOP2 targeting chemotherapeutics is regulated by Oct1 and FILIP1L. PLoS One. 2012; 7:e42921.

41. Filtz EA, Emery A, Lu H, Forster CL, Karasch C, Hallstrom TC. Rb1 and Pten Co-Deletion in Osteoblast Precursor Cells Causes Rapid Lipoma Formation in Mice. PLoS One. 2015; 10:e136729.

42. Lu H, Liang X, Issaenko OA, Hallstrom TC. Jab1/CSN5 mediates E2F dependent expression of mitotic and apoptotic but not DNA replication targets. Cell Cycle. 2011; 10:1-10. 